\title{
THE ASSESSMENT AND SPECIFICITY OF ENVIRONMENTAL AND GENOTYPE-ENVIRONMENTAL COMPONENTS OF VARIABILITY
}

\author{
JEAN M. PERKINS and J. L. JINKS \\ Department of Genetics, University of Birmingham, P.O. Box 363, \\ Birmingham B15 2TT, England
}

Received 1.iv.72

\begin{abstract}
SuMMARY
The statistical and biometrical genetical advantages and disadvantages of using dependent and independent assessments of the environmental values in the joint regression analysis of genotype-environmental interactions have been investigated. The material consisted of 82 inbred lines produced by nine successive generations of selfing from a random sample of $F_{2}$ individuals from the cross between varieties 1 and 5 of Nicotiana rustica, these two varieties and their $F_{1}$. All were grown in the eight environments produced by two planting densities in each of four sowing dates. Ten of these inbred lines, chosen to be a stratified sample of the 82 lines for the character final height, were grown in the 16 environments produced by all combinations of presence and absence of $\mathrm{N}, \mathrm{P}, \mathrm{K}$ and $\mathrm{Ca}$ fertilisers. Eight individually randomised plants of each family were grown in each environmental treatment and flowering time, linear growth rate, leaf length and final height recorded. In addition to the usual dependent assessment of the environments, the main experiment provided three sources of independent assessment, namely, replicate samples of individuals of each inbred line, replicate samples of inbred lines and the parental varieties 1 and 5 . As far as the significance of the heterogeneity of regression and remainder items in the joint regression analysis and the ranking of the inbred lines on the basis of their linear regression coefficients are concerned, it made little difference whether the dependent or any one of the three independent measures of the environmental values was used. The independent measures, however, raised statistical problems where these were based on few observations and problems of interpretation at the biometrical genetical level. Epistatic components of mean performance over all environments for flowering time, of linear sensitivity to environmental differences for growth rate and of non-linear sensitivity for final height have been detected. The correlations over lines between mean performance and linear sensitivity were generally low and non-significant and the number of effective factors of the largely independent genetical systems controlling these two aspects of the phenotype have been estimated. The stratified sample of 10 inbred lines, on the other hand, showed a positive correlation between mean performance and linear sensitivity in the very poor environments produced by the $\mathrm{N}, \mathrm{P}$ and $\mathrm{K}$ treatments in the absence of calcium. This is expected since the performances of all the lines must ultimately converge as they approach the worse environments and during convergence these two aspects of the phenotype must be correlated. The relative linear sensitivities of these lines to the different kinds of environmental variables revealed a high degree of specificity.
\end{abstract}

\section{INTRODUCTION}

Freeman And Perkins (1971) have drawn attention to the problems that can arise from using dependent measures on the environmental values $\left(\hat{\epsilon}_{j}\right)$ for partitioning the genotype-environmental component of variability of family means over environments, into its linear and non-linear portions 
using the analytical procedures first proposed by Yates and Cochran (1938). They also outlined a number of experimental designs that overcame these problems by providing independent measures of the environmental values $\left(\hat{z}_{j}\right)$ and discussed the criteria that must be met for these to be adequate. Comparisons of the use of dependent and independent environmental values (the latter including both biological and physical measures) for analysing genotype-environmental interactions have been made with the fungus Schizophyllum commune (Fripp and Caten, 1971; Fripp, 1972). Parallel studies with the flowering plant Nicotiana rustica are reported in this paper.

\section{Material}

The material consists of 82 inbred lines derived by the authors from nine successive generations of selfing from single, randomly chosen, $\mathrm{F}_{2}$ plants of the cross made between varieties 5 and 1 of $\mathcal{N}$. rustica together with the two parental varieties, 5 and 1 , and their $F_{1}$. This cross and its derivartives were chosen because it is the most thoroughly investigated of those available. From the analysis of many generations derived from this cross, since the initial investigations of Mather and Vines (1952), the genetical systems controlling the mean phenotype of a number of characters and their environmental sensitivities have been shown to be relatively simple (Bucio Alanis, 1966; Bucio Alanis, Perkins and Jinks, 1969; Jinks and Perkins, 1969; Perkins and Jinks, 1970). A number of combinations of genotypes are also available in this material which will give meaningful independent environmental measures of the kind suggested by Freeman and Perkins (1971).

\section{ENVIRONMENTS AND EXPERIMENTAL DESIGN}

Eight environments were produced from the eight combinations of two densities in each of four sowings. The densities consisted of the standard planting density for all $\mathcal{N}$. rustica experiments at Birmingham University, i.e. $70 \mathrm{~cm}$. between rows and $30 \mathrm{~cm}$. within rows, and a high density of $30 \mathrm{~cm}$. both between and within rows. The four successive sowings were at fortnightly intervals between 21 st April and 2nd June 1971. In each of these eight sowing date-density combinations, eight individually randomised plants for each of the 82 inbred lines, varieties 5 and 1 and their $F_{1}$, were grown.

In addition, 10 inbred lines were chosen from the 82 as a stratified sample for the character final height and were grown at a high density $(25 \mathrm{~cm}$. between and within rows) in each of 16 fertiliser environments consisting of all combinations of the presence and absence of N, P, K and Ca. As for the larger experiment, each line was represented by eight individually randomised plants in each environment.

In this paper, both the combinations of sowing dates and densities and of $\mathrm{N}, \mathrm{P}, \mathrm{K}$ and $\mathrm{Ca}$ have been used merely to generate macro-environmental differences. The experimental design, however, allows further orthogonal comparisons to be made among both kinds of environmental variables and for their interaction with genotypes, but such analyses will be the subject of a future paper.

The plants were scored for the following four characters:

(i) Flowering time, FT, as days after sowing. 
(ii) Linear growth rate, GR, measured as the difference between two plant heights (in centimetres) taken at the beginning and end of the flowering period, divided by the number of intervening days.

(iii) Leaf length, LL, in centimetres. The length of the fully expanded 8 th leaf was measured at the commencement of flowering.

(iv) Final height, FH, in centimetres.

An extensive survey was made of a large number of characters in this material by Eaves and Brumpton (1972). They showed that at both the genotypic and genotype-environmental interaction levels characters typified by flowering time, growth rate and final height were correlated and generally independent of characters determining leaf morphology.

\section{RESUlts FOR THE TWO PARENTS, THE $F_{1}$ AND THE 82 INBREd LINES}

In table 1 the mean, $\hat{m}$, and the additive, $[\hat{d}]$, and the dominance, $[\hat{h}]$, genetical components have been estimated from the average performance

TABLE 1

The mean, $\hat{m}$, and additive, $[\hat{d}]$, and dominance, $[\hat{h}]$, genetical components for flowering time, $F T$, groweth rate, GR, leaf length, LL, and final height, FH, (see Section 3) of the parental varieties 5 and 1 and their $F_{1}$ when averaged over the eight sowing date-density combinations

\begin{tabular}{|c|c|c|c|c|}
\hline \multirow[b]{2}{*}{ Component } & \multicolumn{4}{|c|}{ Character } \\
\hline & $\mathrm{FT}$ & GR & LL & $\mathrm{FH}$ \\
\hline $\begin{array}{c}\hat{m} \\
{[\hat{d}]} \\
{[\hat{h}]}\end{array}$ & $\begin{array}{c}75.875 \\
1.344 \pm 0.224 \\
-0.875 \pm 0.548 \dagger\end{array}$ & $\begin{array}{c}3 \cdot 346 \\
0 \cdot 376 \pm 0 \cdot 030 \\
0.547 \pm 0 \cdot 064\end{array}$ & $\begin{array}{c}15 \cdot 262 \\
-0.113 \pm 0 \cdot 166 \dagger \\
2 \cdot 223 \pm 0.309\end{array}$ & $\begin{array}{r}110 \cdot 625 \\
7 \cdot 313 \pm 0 \cdot 796 \\
15 \cdot 094 \pm 1 \cdot 520\end{array}$ \\
\hline
\end{tabular}

over environments of the two parents $\left(P_{1}=\right.$ variety 5 and $P_{2}=$ variety 1$)$ and their $F_{1}$ over all environments assuming that this simple model is adequate (Mather and Jinks, 1971). From the sign of [d] the table shows that $\mathrm{P}_{1}$ flowers later, grows more rapidly and is taller than $\mathrm{P}_{2}$. The genes controlling leaf length are probably completely dispersed between the parents since $[\hat{d}]$ for this character is non-significant. There is a directional component of dominance, $[\hat{h}]$, for faster growth, longer leaves and tallness, but a significant directional component was not found for flowering time.

In an analysis of variance of the parents and $F_{1}$ in each environment the genotype-environmental interaction item was non-significant for all four characters of both the parents and $\mathrm{F}_{1}$, with one exception. A non-linear interaction was detected for the flowering time of the $F_{1}$ (Bucio Alanis, Perkins and Jinks, 1969).

In the absence of scalar effects, non-allelic interactions and selection, the mean of the parents, varieties 5 and 1 , over environments is not expected to differ significantly from that of the inbred lines derived by successive generations of selfing from the $F_{2}$ of the cross between them. The mean of the two parents and of their inbred lines over environments are given in table 2 for each character along with the difference between their two means, the standard deviation of the difference and the significance of the difference from zero. The difference is non-significant for all characters except 
TABLE 2

The parental mean, $\bar{P}$, and the mean of the 82 inbred lines, $L$, when averaged over the eight sowing date-density combinations for the four characters $F T, G R, L L$ and FH (see Section 3)

\begin{tabular}{|c|c|c|c|c|}
\hline \multirow[b]{2}{*}{ Item } & \multicolumn{4}{|c|}{ Character } \\
\hline & FT & GR & LL & FH \\
\hline $\bar{P}$ & $75 \cdot 875$ & $3 \cdot 346$ & $15 \cdot 262$ & $110 \cdot 625$ \\
\hline$L$ & $80 \cdot 100$ & $3 \cdot 266$ & $15 \cdot 293$ & $115 \cdot 703$ \\
\hline Difference & $-4 \cdot 225$ & $0 \cdot 080$ & -0.031 & -5.078 \\
\hline $\begin{array}{l}\text { Standard deviation of } \\
\text { difference } †\end{array}$ & 1.694 & $0 \cdot 160$ & 0.428 & 4.504 \\
\hline Probability & $*$ & n.s. & n.s. & n.s. \\
\hline
\end{tabular}

flowering time. The sign of the difference for flowering time is negative since, on average, the inbred lines flower later than the parents. Because no systematic relationship could be found between the mean and variance of the inbred lines (correlation equals 0.13 ) a scalar effect is not likely to explain the significance of the difference. Neither is selection since, although 18 inbred lines were lost during the inbreeding process, they were, on average, among the later flowering lines. The presence of non-allelic interaction is, therefore, the likeliest explanation. Taking the simplest case of interactions between pairs of homozygous genes, represented by [i] (Jinks and Morley Jones, 1958), the expectation for the parental mean is $m+[i]$ and for that of the inbred lines, $m$. The difference, $-4 \cdot 2251$, is therefore an estimate of the epistatic component, [i].

The estimates of the $\sigma^{2}$ 's for genotypes, $\sigma_{g}^{2}$, environments, $\sigma_{e}^{2}$, genotypes $\times$ environments, $\sigma_{g \times e}^{2}$, and within genotypes and environments (between individuals), $\sigma_{w}^{2}$, as derived from an analysis of variance of the 82 inbred lines over environments are given in table 3 for each character. A mixed model in which genotypes are random and environments fixed is appropriate unlike the model used by the authors in previous publications, e.g. Perkins and Jinks (1968a), in which both genotypes and environments are fixed. The two main effects and their interaction are highly significant for all characters. The genotype-environmental interaction component of variation, $\sigma_{g \times e}^{2}$, is however consistently the smallest.

The genotype-environmental interactions of the 82 inbred lines were investigated for linearity by regressing their performance in each environment

TABLE 3

Estimates of the $\sigma^{2}$ 's from a two-way analysis of variance of the 82 inbred lines in the eight sowing date-density combinations (with replicate individuals) for the four characters FT, GR, LL and FH (see Section 3)

\[ \sigma^{2} \]
Genotypes, $\sigma_{g}^{2}$
Environments, $\sigma_{e}^{2}$
Genotypes $\times$ environments, $\sigma_{g \times e}^{2}$
Within genotypes and
environments, $\sigma_{w}^{2}$

\begin{tabular}{rccr}
\multicolumn{4}{c}{ Character } \\
FT & GR & LL & FH \\
34.87 & 0.19 & $2 \cdot 10$ & 173.72 \\
6.44 & 0.15 & 0.18 & 133.53 \\
3.11 & 0.05 & 0.17 & 17.31 \\
21.22 & 0.23 & 4.11 & 115.49
\end{tabular}


against a biological measure of the environments. For comparative purposes, four different kinds of material were used to assess these environments (Freeman and Perkins, 1971). They are given in descending order of relationship to the 82 inbred lines whose interactions are to be investigated.

\section{(a) Dependent $\hat{\epsilon}_{j}$}

The performance of each of the 82 inbred lines was regressed against the mean of all 82 lines in each environment, i.e. the material used for the environmental assessment is the same as that to be investigated.

\section{(b) Independent $\hat{z}_{j}$ using replicate individuals}

Each inbred line in each environment was represented by eight individual plants. These were split at random into two groups of four, the interactions of one group to be investigated and the other group contributing to the environmental assessment.

\section{(c) Independent $\hat{z}_{j}$ using replicate sets of inbred lines}

The 82 inbred lines were divided at random into two sets of 41 , the interactions of one set to be investigated and the other set to assess the environment.

\section{(d) Independent $\hat{z}_{j}$ using parents}

The 82 inbred lines were regressed against the average of the two parents, varieties 5 and 1 , in each environment from whose $F_{2}$ they were derived by selfing.

Groups $(b)$ and $(c)$ were further divided into subgroups $(b)$ i and $(b)$ ii and $(c)$ i and $(c)$ ii. Subgroups $(b) \mathrm{i}$ and $(b)$ ii represent the regression of the 82 inbred lines in one set of replicate individuals against the mean of the other set in each environment and then vice versa. Similarly, subgroups $(c) i$ and $(c)$ ii represent the regression of the 41 inbred lines in one set against the mean of the other set in each environment and then vice versa. For comparative purposes the 41 lines in each set were also regressed against their own dependent environmental measures, $\hat{\epsilon}_{j}$ 's, in separate analyses.

The adequacy with which the environments are assessed depends upon the degree of relationship between the genotypes whose interactions are to be investigated and the genotypes used to assess the environment and also upon the purposes for which the genotype-environmental interaction assessments are required. If a mere ranking of the genotypes is required according to the magnitude of their linear regression coefficients, $\bar{\beta}+\hat{\beta}_{d}$ 's (when derived from the regression of a genotypic performance in each environment against an environmental assessment), it is only necessary for the joint regression item to be significant when tested against the joint remainder. If, however, genetical interpretations of their linear genotype-environmental interactions are to be made, two further criteria must be satisfied (Freeman and Perkins, 1971). The joint remainder should be non-significant when tested against the variance within genotypes and environments (between individuals) and the joint regression coefficient, $\bar{\beta}$, should not be significantly different from one. In table 4 the results of applying these two criteria to the joint regression analyses of the four characters of the inbred lines against the three kinds of independent environmental assessors, $(b),(c)$ and $(d)$, are given. The table shows that, with the exception of leaf length, the only 
independent assessment of the environment which consistently satisfies both criteria is a replicate set of individuals, $(b)$ i and $(b)$ ii. A replicate set of inbred lines, $(c) \mathrm{i}$ and $(c)$ ii, is satisfactory to the extent that the joint regression coefficient, $\tilde{\beta}$, is never significantly different from one.

In column $(d) \mathrm{i}$ the results of applying the two criteria to the joint regression against the independent environmental assessor (as in $(b) \mathrm{i}$ and $(b)$ ii and $(c)$ i and $(c)$ ii) are given. According to these results, the use of the parents (varieties 5 and 1 ) to assess the environment consistently fails on both tests. However, the average of the parents is based upon fewer observations than the average of the 82 inbred lines in each environment. In this case therefore, unlike $(b)$ and $(c)$, the material used as the independent variate in

TABLE 4

Testing the adequacy of the independent environmental assessors $(b) i,(b) i i,(c) i,(c) i i,(d) i$ and $(d) i i$ (see Section 4) for the four characters FT, GR, LL and FH (see Section 3) from the significance of the joint regression, $\bar{\beta}$, from one and of the joint remainder

\begin{tabular}{|c|c|c|c|c|c|c|c|}
\hline \multirow[b]{2}{*}{ Character } & \multirow[b]{2}{*}{ Item } & \multicolumn{6}{|c|}{ Independent environmental assessor } \\
\hline & & (b) $\mathrm{i}$ & (b) $\mathrm{ii}$ & (c) $\mathbf{i}$ & (c) $\mathrm{ii}$ & (d) $\mathrm{i}$ & (d) $\mathrm{ii}$ \\
\hline \multirow[t]{3}{*}{ FT } & $\beta$ & 0.96 & $1 \cdot 02$ & $1 \cdot 08$ & 0.89 & 0.73 & $1 \cdot 32$ \\
\hline & Significance of $\beta-1$ & n.s. & n.s. & n.s. & n.s. & $* *$ & $* * *$ \\
\hline & $\begin{array}{l}\text { Significance of joint } \\
\text { remainder }\end{array}$ & n.s. & n.s. & $*$ & n.s. & $* * *$ & n.s. \\
\hline \multirow[t]{3}{*}{ GR } & $\bar{\beta}$ & $0 \cdot 97$ & $1 \cdot 02$ & $1 \cdot 04$ & $1 \cdot 01$ & 0.62 & $1 \cdot 58$ \\
\hline & Significance of $\beta-1$ & n.s. & n.s. & n.s. & n.s. & $* * *$ & $* * *$ \\
\hline & $\begin{array}{l}\text { Significance of joint } \\
\text { remainder }\end{array}$ & n.s. & n.s. & $*$ & $*$ & $* * *$ & n.s. \\
\hline \multirow[t]{3}{*}{ LL } & $\beta$ & $0 \cdot 70$ & $1 \cdot 25$ & $1 \cdot 03$ & 0.73 & 0.44 & $1 \cdot 24$ \\
\hline & Significance of $\beta-1$ & $* * *$ & n.s. & n.s. & n.s. & $*$ & n.s. \\
\hline & $\begin{array}{l}\text { Significance of joint } \\
\text { remainder }\end{array}$ & n.s. & $*$ & $* * *$ & $* *$ & $* * *$ & n.s. \\
\hline \multirow[t]{3}{*}{ FH } & $\bar{\beta}$ & $1 \cdot 02$ & $0 \cdot 97$ & 0.99 & $1 \cdot 01$ & 0.81 & $1 \cdot 17$ \\
\hline & Significance of $\beta-1$ & n.s. & n.s. & n.s. & n.s. & $\begin{array}{l}\text { n.s. } \\
* * *\end{array}$ & $\begin{array}{c}\text { n.s. } \\
*\end{array}$ \\
\hline & $\begin{array}{l}\text { Signiticance of joint } \\
\text { remainder }\end{array}$ & & & & & & \\
\hline & $\begin{array}{r}\text { n.s. } \\
* \\
* * \\
* *\end{array}$ & $\begin{array}{l}\text { abil } \\
\text { abil } \\
\text { abil } \\
\text { abil }\end{array}$ & $\begin{array}{l}\text { non- } \\
0.01 \\
0.00 \\
<0 .\end{array}$ & $\begin{array}{l}\text { ificant. } \\
1 .\end{array}$ & & & \\
\hline
\end{tabular}

assessing the environment is subject to a greater sampling variance than the inbred lines used as the dependent variate in the joint regression. In column (d) ii the average, in each environment, of the parents (which has the greater error) has been regressed against the average, in each environment, of the 82 inbred lines. It is clear that the number of significant tests has been reduced. The joint regression, $\bar{\beta}$, is still significantly different from one, however, for flowering time and growth rate and the joint remainder is significant for final height. It is possible, therefore, as similarly found by Fripp (1972), to obtain misleading results for the joint regression when the environmental measure used as the independent variate is based upon far fewer observations than the genotypes constituting the dependent variate.

In table 5 the significance of the heterogeneity of regressions and of the heterogeneity of remainders in the joint regression analyses of the inbred lines against the four different kinds of environmental assessors, $(a),(b),(c)$ and $(d)$, are given for each character. The heterogeneity of remainders was 
tested against the variance within genotypes and environments (between individuals). The heterogeneity of regressions was tested against the heterogeneity of remainders if the latter was significant, if not, the heterogeneity of regressions was tested against the variance within genotypes and environments. The results are completely consistent across all the different ways of assessing the environment with the exception, again, of leaf length. Thus for flowering time there are significant non-linear but no linear genotypeenvironmental interactions. For both growth rate and final height there are significant linear and non-linear interactions. A large sampling variance has repeatedly made itself evident for leaf length. Thus, for example, in table 3 the $\sigma^{2}$ for the variance within genotypes and environments, $\sigma_{w}^{2}$, has proportionately the largest value among those for leaf length compared with the other three characters. The tests for the linear and non-linear interactions (table 5) are so insensitive for leaf length that the interaction becomes undetectable when the number of replicate individuals is halved in order to provide an independent assessment of each environment as in $(b) \mathrm{i}$ and $(b) \mathrm{ii}$.

TABLE 5

Significances of the heterogeneity of regression and of the heterogeneity of remainder for the four characters $F T, G R, L L$ and FH (see Section 3) using the environmental assessors $(a),(b) i,(b) i i,(c) i,(c) i i$ and $(d)$ (see Section 4)

Environmental assessor

\begin{tabular}{clcccccc} 
Character & \multicolumn{1}{c}{ Item } & $(a)$ & $(b) \mathrm{i}$ & $(b) \mathrm{ii}$ & $(c) \mathrm{i}$ & $(c)$ ii & $(d)$ \\
FT & Heterogeneity of regression & n.s. & n.s. & n.s. & n.s. & n.s. & n.s. \\
& Heterogeneity of remainder & $* * *$ & $* * *$ & $* * *$ & $* * *$ & $* * *$ & $* * *$ \\
GR & Heterogeneity of regression & $* * *$ & $* * *$ & $* * *$ & $* * *$ & $* * *$ & $* * *$ \\
& Heterogeneity of remainder & $* * *$ & $* * *$ & $* * *$ & $* * *$ & $* * *$ & $* * *$ \\
LL & Heterogeneity of regression & $* * *$ & n.s. & n.s. & $* *$ & $* *$ & n.s. \\
& Heterogeneity of remainder & $* *$ & n.s. & n.s. & $* *$ & n.s. & $* *$ \\
FH & Heterogeneity of regression & $* * *$ & $* * *$ & $* * *$ & $* * *$ & $* * *$ & $* * *$ \\
& Heterogeneity of remainder & $* * *$ & $* * *$ & $* * *$ & $* * *$ & $* * *$ & $* * *$ \\
& \multicolumn{2}{c}{ n.s., **, $* * *=$ Probability as in table 4. } & & &
\end{tabular}

When the genotypes are regressed for leaf length against the other sources of environmental assessment there is some evidence for both a linear and nonlinear component of the genotype-environmental interactions.

The rank correlations (Spearman, 1904) over the 82 inbred lines between the linear regression coefficient, $1+\widehat{\beta}_{d}$, obtained with the dependent environmental component, $\hat{\epsilon}_{j}$, and the corresponding coefficient, $\bar{\beta}+\hat{\beta}_{d}$, obtained with each kind of independent environmental component, $\hat{z}_{j}$, are given in table 6 for all characters. On the basis of these correlations there is little to choose between the different kinds of environmental assessment since all are highly significant $(\mathrm{P}<0.00 \mathrm{l})$ and, with the exception of leaf length, all have very high values.

\section{ENVTRONMENTAL ASSESSMENT}

The results given in tables 5 and 6 show that, in general, there is nothing to choose between the dependent, $\hat{\epsilon}_{j}$, and an independent, $\hat{z}_{j}$, environmental component in assessing the environment as far as either the significances of the heterogeneity of regressions and of remainders or the ranking of the genotypes on the basis of their linear regression coefficients is concerned. It 
is therefore questionable whether, in experiments with $\mathcal{N}$.rustica, it is worth dividing the resources available to provide separate assessments of both the interactions and of the environment, to the detriment of both. Thus no genotype-environmental interactions were detected for leaf length, known to have a relatively large sampling variance, when the number of replicate individuals was halved in order to provide an independent environmental assessment. The danger of deriving an independent environmental assessment based upon the average of too few genotypes in each environment was also demonstrated by the joint regression analyses in Section 4 between the 82 inbred lines and the two parental varieties (see also Fripp, 1972).

When biometrical genetical interpretations are required, the use of an independent environmental assessment will also be inadequate if the genotypes used to assess the environment have on average different genotypeenvironmental interaction expectations to those of the genotypes to be investigated because of differences in gene frequencies or in gene action at the relevant loci. Although there is evidence of an epistatic component, [i],

TABLE 6

Rank correlations for the four characters FT, GR, LL and FH (see Section 3) over the 82 inbred lines between their regression coefficients with the dependent environmental assessment, $1+\hat{\beta}_{d}$, and those, $\bar{\beta}+\hat{\beta}_{d}$ 's, with the different kinds of independent environmental assessments, $(b) i$, (b) $i i,(c) i,(c)$ ii and $(d)$ (see Section 4)

\begin{tabular}{ccccc} 
Independent & \multicolumn{4}{c}{ Character } \\
\cline { 2 - 5 } environmental assessors & FT & GR & LL & FH \\
$(b)$ i & 0.836 & 0.934 & 0.665 & 0.929 \\
$(b)$ ii & 0.969 & 0.926 & 0.810 & 0.901 \\
$(c)$ i† & 0.950 & 0.995 & 0.911 & 0.995 \\
$(c)$ ii & 0.940 & 0.982 & 0.844 & 0.997 \\
$(d)$ & 0.974 & 0.977 & 0.708 & 0.976
\end{tabular}

$\dagger$ The rank correlations are in general based upon 80 degrees of freedom but those for $(c) \mathrm{i}$ and $(c)$ ii are based upon 39 .

for flowering time which makes a different contribution to the mean performance of the genotypes under investigation and the two parental varieties used to assess the environments (table 2), this does not, of itself, invalidate the use of the parental varieties since [i] is a constant in all environments. The average sensitivity of the parental varieties and of the inbred lines to environmental differences are expected to be the same if additive gene action alone is involved in the genotype-environmental interactions. The presence of an epistatic component of this interaction would, therefore, invalidate the use of the parental varieties. Providing, therefore, that selection has not led to any deviation from equal gene frequencies among the 82 surviving inbred lines, any inadequacy of the parental varieties for assessing the environments can be attributed to an epistatic component of the genotype-environmental interactions. Reference to column $(d)$ ii of table 4 suggests that the parental varieties are, in fact, inadequate for three out of the four characters. Thus for final height only the joint remainder item in the joint regression analysis is significant on the more conservative test of regressing the mean of the two parents on to the mean of the 82 inbred lines in each environment. This implies that the mean of the parents differs significantly from the mean of the inbred lines in some environments but that 
the magnitude of the difference is not correlated with the environmental component, $\hat{\epsilon}_{j}$. Conversely, for flowering time and growth rate the joint regression coefficient, $\bar{\beta}$, is significantly greater than 1 while the joint remainder is non-significant. Thus in those environments in which the inbred lines have an extreme high or low performance for flowering time or growth rate the parental varieties are even more extreme in their performance for these characters.

\section{Biometrical genetical interpretations}

In a previous paper we have shown that the large positive correlations between mean performance and sensitivity to environmental variation (both linear and non-linear), which are observed in $\mathcal{N}$. rustica in common with many other species, are reduced following opportunities for re-assortment and re-combination in the generations derived from an initial cross between pairs of lines which show this correlation (Perkins and Jinks, 1968a). We have also shown that the sensitivity to the macro-environmental differences produced by deliberately imposed treatments and the sensitivity to microenvironmental variation within these treatments due to uncontrolled environmental differences are uncorrelated in some varieties of $\mathcal{N}$. rustica and the generations derived from crosses between them (Perkins and Jinks, $1968 a, b$ and $1971 b$ ). Thus, even though these three aspects of the phenotype, namely, mean performance, sensitivity to macro-environmental differences and sensitivity to micro-environmental variation, are generally correlated, there is evidence that they are controlled, at least in part, by independent genetical systems. The 82 inbred lines provide a larger population than has hitherto been available for re-examining the interdependence of these systems.

The linear regression coefficient, $\hat{\beta}_{\boldsymbol{d}}$, obtained with the dependent environmental component, $\hat{\epsilon}_{j}$, will be used as the measure of linear sensitivity of the 82 inbred lines to macroenvironmental differences. For flowering time the corresponding heterogeneity of regression is not significant against the remainder mean square (table 5, column $(a)$ ) although it is significant against the variance within genotypes and environments. The correlations for flowering time which involve this component have, therefore, been included in this discussion since, in some instances, their signs and significances are consistent with those obtained for the other characters.

The correlations over the 82 inbred lines between the measures of their relative mean performances (the additive genetical component, $[d]$ ) and their linear sensitivities to macro-environmental differences (the linear interaction coefficient, $\hat{\beta}_{d}$ ) are $-0.689,0.071,-0.006$ and 0.135 for flowering time, growth rate, leaf length and final height respectively. Thus, apart from the indication of a negative value for flowering time, the magnitudes of the correlations for the three remaining characters are very small and confirm that these two aspects of the phenotype are under independent genetical control.

Rank correlations over the 82 inbred lines for 80 degrees of freedom between the average variance within environments, $\hat{\sigma}_{w}^{2}$, and the linear regression coefficient, $\hat{\beta}_{d}$, and the total variance over environments, $\hat{V}_{G+E}$, which are, respectively, a measure of sensitivity to micro-environmental variation, of linear sensitivity to macro-environmental differences and of 
total sensitivity (linear and non-linear) to macro-environmental differences, are given in table 7. For flowering time and growth rate, the rank correlation between the $\hat{\beta}_{d}$ 's and the $\hat{\sigma}_{w}^{2}$ 's is significantly negative, i.e. the greater the linear sensitivity to environmental differences, the smaller the sensitivity to micro-environmental variation. For leaf length there is a significant positive correlation between the rankings of the total variance over environments, $\hat{V}_{G+E}$, and the average variance within environments, $\hat{\sigma}_{w}^{2}$. The rank correlations for all other characters are very small and non-significant. Hence,

TABLE 7

The rank correlations over the 82 inbred lines between the average variance within environments, $\hat{\sigma}_{w}^{2}$, and the linear regression coefficient, $\hat{\beta}_{d}$ (with the dependent environmental component, $\left.\hat{\epsilon}_{j}\right)$, and the total variance between environments, $\bar{V}_{G+E}$, for the four characters, FT, GR, LL and $F H$ (see Section 3)

\begin{tabular}{|c|c|c|c|c|}
\hline \multirow{3}{*}{$\begin{array}{l}\text { Correlation of } \\
\hat{\beta}_{a} \text { and } \hat{\sigma}_{w}^{2} \\
\hat{\nabla}_{G+E} \text { and } \hat{\sigma}^{2}\end{array}$} & \multicolumn{4}{|c|}{ Character } \\
\hline & FT & GR & LL & $\mathrm{FH}$ \\
\hline & $\begin{array}{r}-0.581 * * * \\
0.073 \text { n.s. }\end{array}$ & $\begin{array}{l}-0.309 * * \\
-0.143 \text { n.s. }\end{array}$ & $\begin{array}{l}0.213 \text { n.s. } \\
0.324^{* *}\end{array}$ & $\begin{array}{l}-0.209 \text { n.s. } \\
-0.151 \text { n.s. }\end{array}$ \\
\hline
\end{tabular}

there is a fair degree of independence in the genetical control of sensitivity at the micro- and macro-environmental levels.

Estimates of the number of effective factors controlling the differences among the lines for the additive genetical component and for the linear regression coefficient (Perkins and Jinks, 1968b; Mather and Jinks, 1971; Eaves and Brumpton, 1972) are given in table 8. These are minimal estimates for the usual reasons. In addition, the estimate for the additive

TABLE 8

Number of effective factors, $\hat{k}$, controlling the differences among the 82 inbred lines in mean performance, as measured by the additive genetical component $[\hat{d}]$, and its linear sensitivity to macro-environmental differences, as measured by the regression coefficient, $\hat{\beta}_{d}$ (with a dependent environmental component, $\left.\hat{\epsilon}_{j}\right)$, for the four characters $F T, G R, L L$ and $F H$ (see Section 3)

\begin{tabular}{|c|c|c|c|c|}
\hline \multirow[b]{2}{*}{$k$ for } & \multicolumn{4}{|c|}{ Character } \\
\hline & FT & GR & LL & $\mathrm{FH}$ \\
\hline$[\hat{d}]$ & 7 & 7 & 9 & 5 \\
\hline$\hat{\beta}_{d}$ & 0 & 8 & 14 & 8 \\
\hline
\end{tabular}

component for flowering time is further minimised by the presence of epistasis (Section 4) while the estimate for the linear sensitivity of this character is recorded as zero because its heterogeneity of regression was non-significant (table 5). The estimates for the additive genetic component are almost identical with the independent estimates of Eaves and Brumpton (1972). It is clear from the earlier publications and from the substantive evidence of the present paper that the majority of the effective factors involved in the control of the mean performance for flowering time, growth rate and final height are common to all three characters, but this will be pursued in a later paper. It is equally clear from the absence of significant correlations noted earlier in this section that there are few, if any, effective factors acting 
in common upon both relative mean performance $([\hat{d}])$ and linear sensitivity to macroenvironmental differences $\left(\hat{\beta}_{d}\right)$.

\section{RESULTS FOR THE 10 INBRED LINES}

The 10 inbred lines are a stratified sample of the 82 lines, described in Section 4, for the character final height. Genotypes in this section, therefore, are treated as a fixed effect together with the environments in the analysis of variance and the regressions have been made against the dependent environmental component, $\hat{\epsilon}_{j}$. The eight combinations of nitrogen, phosphorus and potassium are treated separately in the absence and presence of calcium because of the large and obvious difference for all the characters between these two sets (Perkins and Jinks, 1971 a). The performances of the 10 inbred lines in the eight combinations of the four sowing dates and two density levels have not been separated since a significant overall difference between

TABLE 9

Significances of the heterogeneity of regressions and of the heterogeneity of remainders for the characters FT, GR, LL and FH (see Section 3) of the 10 inbred lines in the three environmental sets, NPK, NPKCa and $S-D$ (see Section 7), using the dependent environmental assessor, $\hat{\epsilon}_{j}$

\begin{tabular}{|c|c|c|c|c|}
\hline \multirow[b]{2}{*}{ Character } & \multirow[b]{2}{*}{ Item } & \multicolumn{3}{|c|}{ Environmental Set } \\
\hline & & NPK & NPKCa & S-D \\
\hline FT & $\begin{array}{l}\text { Heterogeneity of regression } \\
\text { Heterogeneity of remainder }\end{array}$ & ** & $\begin{array}{l}* * * \\
\text { n.s. }\end{array}$ & $\begin{array}{l}\text { n.s. } \\
* * *\end{array}$ \\
\hline GR & $\begin{array}{l}\text { Heterogeneity of regression } \\
\text { Heterogeneity of remainder }\end{array}$ & *** & $* *$ & ** \\
\hline LL & $\begin{array}{l}\text { Heterogeneity of regression } \\
\text { Heterogeneity of remainder }\end{array}$ & *** & $\begin{array}{l}\text { n.s. } \\
\text { n.s. }\end{array}$ & $\begin{array}{l}\text { n.s. } \\
\text { n.s. }\end{array}$ \\
\hline FH & $\begin{array}{l}\text { Heterogeneity of regression } \\
\text { Heterogeneity of remainder }\end{array}$ & ** & $\begin{array}{l}* * * \\
* * *\end{array}$ & $\begin{array}{c}\text { n.s. } \\
* *\end{array}$ \\
\hline
\end{tabular}

densities has only been obtained for growth rate and final height (Perkins, unpublished) and even then the magnitude of the difference is smaller than that obtained between the two sets of fertiliser environments, with and without calcium.

For each of the four characters analyses of variance have been carried out for the 10 inbred lines in each of the three sets of environments, the latter being the eight fertiliser combinations without calcium, NPK, the eight combinations with calcium, NPKCa, and the eight combinations of sowing dates and densities, S-D. The items for genotypes, environments and genotypes $\times$ environments in these analyses are significant for all characters in all environmental sets except for the interaction item of leaf length in the fertiliser combinations with calcium and the sowing date-density combinations.

Corresponding joint regression analyses have been carried out and the results are given in table 9 . Comparison of this table with column (a) of table 5 shows that the significances of the regression items for the 10 and 82 inbred lines, respectively, in the sowing date-density set of environments agree for the characters flowering time and growth rate, but not for leaf length and final height. The non-significance of the heterogeneity of 
regression for final height indicates that while the 10 inbred lines were selected to sample the complete range of mean performances over environments, they do not constitute a similar sample of the range of linear regression coefficients. This is not surprising since the mean performance over environments and the linear regression coefficients of the 82 inbred lines have already been shown to be independent (Section 6). Reference to table 9 leaves no doubt that the incidence of the genotype-environmental interaction and the relative magnitudes of its linear and non-linear components differ markedly over the three sets of environments. This can also be seen from the estimates of the additive genetical component, $[\hat{d}]$, and the linear regression coefficient, $\hat{\beta}_{d}$, of the 10 inbred lines and of the mean, $\hat{m}$, in the three sets of environments given for each character in table 10. For those combinations of characters and environmental sets in which the heterogeneity of regression item in table 9 is non-significant, the corresponding $\hat{\beta}_{d}$ values in table 10 are italicised.

\section{The SPEGIFIGITY OF THE INTERAGTION OF THE 10 LINES WITH THE THREE GONTRASTING SETS OF ENVIRONMENTS}

The obvious differences in the pattern of interactions of the 10 inbred lines in the three sets of environments have been pursued further by analyses of variance and of joint regression in which their performance in all three sets are combined. These showed significant overall differences between sets and between genotypes and a significant interaction between sets and genotypes for all four characters. In the combined joint regression analysis a significant pooled remainder mean square (162 degrees of freedom) was found for all characters except leaf length. The heterogeneity of regression over environmental sets ( 9 degrees of freedom) was significant for all characters except growth rate while both flowering time and growth rate showed a significant interaction of this item with environmental sets (18 degrees of freedom). For flowering time and growth rate, therefore, the pattern of relative linear sensitivities of the 10 inbred lines is specific to the particular environmental set. Reference to table 10 shows that this specificity arises from an almost complete reversal in the ranking of the $\hat{\beta}_{d}$ values between the NPK and sowing date-density set of environments especially for growth rate. It would appear, therefore, that inbred lines which, on average, are faster in growth rate and which in the environments of the sowing date-density set have the lowest linear sensitivity to environmental differences become the most sensitive to such differences in the environments of the NPK set. Equally, the inbred lines which on average, are slower in growth rate have the greatest linear sensitivity in the sowing date-density environments and become the least sensitive in the NPK environments. For the other two characters, final height and leaf length, there is no significant evidence of specificity. Corresponding analyses of flowering time and final height in varieties 2 and 42 of $\mathcal{N}$. rustica grown in contrasting environmental sets, which were based on sowing dates and fertiliser treatments, led to identical conclusions in respect of the specificity of their linear sensitivity to contrasting environmental sets to those reported here (Perkins and Jinks, 1971a).

The mean performance in each environmental set ( $\hat{m}$ of table 10$)$, which differ significantly over sets for all characters, show that plant growth and size as measured by the characters growth rate, leaf length and final height are much reduced in the NPK set and to a lesser extent in the NPKCa set 
TABLE 10

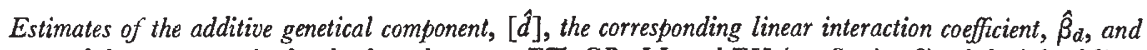
of the set mean, $\hat{m}$, for the four characters $F T, G R, L L$ and $F H$ (see Section 3 ) of the inbred lines in the three environmental sets (see Section 7)

\begin{tabular}{|c|c|c|c|c|c|c|c|}
\hline & & & {$[\hat{d}]$} & & & $\hat{\beta}_{d} \dagger$ & \\
\hline & Line & NPK & NPKCa & S-D & NPK & NPKCa & S-D \\
\hline FT & 1 & $-10 \cdot 37$ & $-8 \cdot 15$ & $-7 \cdot 87$ & -0.74 & -0.88 & 0.28 \\
\hline & 2 & $-7 \cdot 84$ & -5.99 & $-5 \cdot 36$ & -0.54 & -0.27 & -0.23 \\
\hline & 3 & -8.39 & $-6 \cdot 26$ & $-6 \cdot 55$ & -0.64 & -0.98 & 0.11 \\
\hline & 4 & -8.06 & $-6 \cdot 04$ & $-6 \cdot 17$ & -0.70 & -0.54 & 0.08 \\
\hline & 5 & 2.75 & $2 \cdot 07$ & 0.39 & 0.08 & 0.47 & 0.22 \\
\hline & 6 & $7 \cdot 80$ & $3 \cdot 78$ & 4.02 & 0.50 & $0 \cdot 14$ & -0.09 \\
\hline & 7 & $3 \cdot 36$ & $2 \cdot 67$ & $3 \cdot 19$ & $0 \cdot 20$ & -0.08 & -0.01 \\
\hline & 8 & $12 \cdot 08$ & $10 \cdot 87$ & $9 \cdot 95$ & 0.98 & $1 \cdot 30$ & -0.35 \\
\hline & 9 & $2 \cdot 11$ & $2 \cdot 35$ & $3 \cdot 17$ & 0.46 & -0.03 & -0.26 \\
\hline & 10 & $6 \cdot 55$ & $4 \cdot 70$ & $5 \cdot 23$ & 0.38 & 0.87 & 0.27 \\
\hline & $\hat{m}$ & $79 \cdot 12$ & $75 \cdot 96$ & $79 \cdot 58$ & - & - & - \\
\hline GR & 1 & -0.97 & $-1 \cdot 25$ & $-1 \cdot 10$ & -0.34 & -0.25 & 0.43 \\
\hline & 2 & -0.29 & -0.35 & -0.30 & -0.07 & $-0 \cdot 17$ & 0.09 \\
\hline & 3 & -0.49 & -0.70 & -0.45 & -0.36 & -0.06 & 0.39 \\
\hline & 4 & -0.46 & -0.62 & $-0 \cdot 19$ & -0.25 & $0 \cdot 20$ & 0.52 \\
\hline & 5 & 0.29 & 0.35 & 0.25 & 0.09 & $0 \cdot 15$ & -0.03 \\
\hline & 6 & 0.09 & $0 \cdot 16$ & 0.00 & 0.00 & -0.08 & -0.70 \\
\hline & 7 & 0.27 & 0.52 & 0.29 & 0.24 & 0.07 & -0.09 \\
\hline & 8 & 0.42 & 0.45 & $0 \cdot 15$ & $0 \cdot 25$ & $-0 \cdot 15$ & -0.50 \\
\hline & 9 & 0.46 & 0.59 & 0.66 & $0 \cdot 18$ & $0 \cdot 14$ & -0.10 \\
\hline & 10 & 0.66 & 0.84 & 0.67 & $0 \cdot 26$ & $0 \cdot 13$ & -0.01 \\
\hline & $\hat{m}$ & $1 \cdot 64$ & $2 \cdot 42$ & $3 \cdot 35$ & - & - & - \\
\hline LL & 1 & $-1 \cdot 18$ & $-1 \cdot 51$ & $-3 \cdot 19$ & -0.04 & -0.03 & -0.29 \\
\hline & 2 & -0.46 & $-0 \cdot 12$ & -0.59 & 0.05 & 0.19 & -0.11 \\
\hline & 3 & 0.64 & 0.39 & 0.05 & -0.02 & -0.02 & 1.03 \\
\hline & 4 & $0 \cdot 14$ & -0.11 & -0.28 & $0 \cdot 01$ & 0.15 & 0.13 \\
\hline & 5 & 0.39 & $1 \cdot 31$ & 1.79 & $0 \cdot 23$ & 0.23 & -0.05 \\
\hline & 6 & -0.61 & -0.29 & 0.90 & $0 \cdot 15$ & -0.01 & 0.30 \\
\hline & 7 & 0.06 & 0.69 & 0.82 & 0.01 & -0.09 & 0.68 \\
\hline & 8 & -0.29 & -0.95 & -0.95 & -0.15 & -0.09 & -0.67 \\
\hline & 9 & 0.36 & -0.06 & $0 \cdot 61$ & -0.13 & -0.20 & 0.07 \\
\hline & 10 & 0.96 & 0.65 & 0.84 & $-0 \cdot 10$ & -0.13 & -0.91 \\
\hline & $\hat{m}$ & $9 \cdot 55$ & $11 \cdot 75$ & $15 \cdot 23$ & - & - & - \\
\hline FH & 1 & $-20 \cdot 61$ & $-24 \cdot 15$ & $-32 \cdot 49$ & -0.24 & -0.28 & -0.03 \\
\hline & 2 & $-7 \cdot 61$ & $-7 \cdot 34$ & $-12 \cdot 17$ & -0.04 & -0.25 & -0.16 \\
\hline & 3 & $-9 \cdot 08$ & $-11 \cdot 15$ & $-15 \cdot 50$ & -0.18 & -0.39 & -0.20 \\
\hline & 4 & $-9 \cdot 69$ & $-12 \cdot 12$ & $-11 \cdot 11$ & $-0 \cdot 14$ & -0.04 & 0.11 \\
\hline & 5 & $-4 \cdot 58$ & -4.44 & $-3 \cdot 30$ & 0.06 & $0 \cdot 22$ & 0.07 \\
\hline & 6 & $6 \cdot 02$ & $6 \cdot 05$ & $7 \cdot 03$ & -0.09 & 0.07 & -0.47 \\
\hline & 7 & $1 \cdot 50$ & $6 \cdot 70$ & $11 \cdot 61$ & 0.28 & 0.20 & 0.42 \\
\hline & 8 & 13.55 & $17 \cdot 76$ & $15 \cdot 81$ & $0 \cdot 17$ & 0.33 & 0.01 \\
\hline & 9 & $8 \cdot 35$ & $9 \cdot 20$ & $13 \cdot 26$ & 0.05 & $0 \cdot 01$ & 0.12 \\
\hline & 10 & $22 \cdot 14$ & 19.49 & $26 \cdot 86$ & 0.12 & $0 \cdot 11$ & 0.12 \\
\hline & $\hat{m}$ & $67 \cdot 61$ & $80 \cdot 60$ & $117 \cdot 36$ & - & - & - \\
\hline
\end{tabular}

$\dagger$ The linear coefficients, $\hat{\beta}_{d}$ 's, are italicised for those character-environmental set combinations in which the corresponding heterogeneity of regressions item was non-significant in table 7 . 
compared with the sowing date-density set. Using $\hat{m}$ as the measure of the average quality of the environments within a set, reference to tables 9 and 10 shows that the poorer the environments within a set, the more significant are the differences among the linear and non-linear interaction components of the 10 inbred lines. Equally, the poorer the environments the greater the positive relationship between the additive genetical component, $[\hat{d}]$, and the linear regression coefficient, $\hat{\beta}_{d}$, over the inbred lines. These relationships are consistent with the performances of the inbred lines linearly converging more and more, as converge they must, on approaching the worst of all environments.

To complete the investigation of the specificity of the genotypeenvironmental interactions in the three environmental sets, the rank correlations (for 8 degrees of freedom) between $\hat{\sigma}_{w}^{2}$ and $\hat{\beta}_{d}$ and $\hat{\sigma}_{w}^{2}$ and $\hat{V}_{G+E}$, comparable to those of the 82 inbred lines given in table 7 and described in Section 6, have been calculated for the 10 inbred lines in each of the three sets. Their significances are given in table 11 and those of the correlations

\section{TABLE 11}

The rank correlations over the 10 inbred lines between $\hat{\sigma}_{w}^{2}$ and $\hat{\beta}_{d}$ and $\hat{V}_{G+E}$ (see table 7 ) for the four characters FT, GR,LL and FH (see Section 3) in the three environmental sets, $\mathcal{N P K}, \mathcal{N P K C a}$ and $S-D$ (see Section 7)

\begin{tabular}{|c|c|c|c|c|c|}
\hline \multirow{4}{*}{$\begin{array}{l}\text { Correlation of } \\
\hat{\beta}_{d} \text { and } \hat{\sigma}_{w}^{2}\end{array}$} & \multirow{2}{*}{$\begin{array}{c}\text { Environmental } \\
\text { set }\end{array}$} & \multicolumn{4}{|c|}{ naracter } \\
\hline & & FT & GR & LL & FH \\
\hline & 1 & $* * *$ & n.s. & n.s. & n.s. \\
\hline & 2 & $* *$ & n.s. & - & $*$ \\
\hline & 3 & $-\uparrow$ & n.s. & - & - \\
\hline$\hat{V}_{G+E}$ and $\hat{\sigma}_{w}^{2}$ & 1 & $* * *$ & n.s. & n.s. & n.s. \\
\hline & 2 & $* *$ & n.s. & n.s. & $* *$ \\
\hline & 3 & n.s. & n.s. & n.s. & n.s. \\
\hline
\end{tabular}

$\dagger$ The rank correlations involving $\hat{\beta}_{d}$ 's for which the corresponding heterogeneity of regression is non-significant (table 9 ) have been omitted.

n.s., $*, * *, * * *=$ Probability as in table 4 .

involving $\hat{\beta}_{d}$ have again been omitted where the corresponding heterogeneity of regression item was not significant. The few significant rank correlations in this table are all positive and there is perfect agreement, for every combination of character and environmental set, in the significance of corresponding rank correlations between $\hat{\sigma}_{w}^{2}$ and $\hat{\beta}_{d}$ and $\hat{\sigma}_{w}^{2}$ and $\hat{V}_{G+E}$. There is a positive association between sensitivity to micro-environmental variation, $\hat{\sigma}_{w}^{2}$, and the two measures of macro-environmental sensitivity, the linear $\left(\hat{\beta}_{d}\right)$ and the total linear and non-linear $\left(\hat{V}_{G+E}\right)$, for flowering time in the two sets of fertiliser environments ( 1 and 2) and for final height in the fertiliser set with calcium (2). Most of the correlations therefore, as previously found for the 82 inbred lines, are small and insignificant, thus confirming that the sensitivity of genotypes to macro- and micro-levels of environmental variation are largely independent of one another. Significant correlations in some environmental sets and no correlations in others, as pointed out by Fripp and Caten (1973), imply that different combinations of genes must be involved in the reactions of the genotypes to different kinds of environmental stimuli. The consequences for the breeder who wishes to select for a particular level of sensitivity to specific environmental variables is clear and has already been discussed in detail by Perkins and Jinks (197l $a$ ). 


\section{RELATIONS WITH EARLy INVESTIGATIONS}

Varieties 1 and 5 and a large number of diverse generations derived from an initial cross between them have been the subject of a number of investigations, many of which have involved examination of their interaction with environments (Perkins and Jinks, 1968a, $b$; Bucio Alanis, Perkins and Jinks, 1969; Jinks and Perkins, 1971b). Some of the comparisons which are now possible between these earlier investigations and that described in the present paper are particularly informative about the nature and specificity of the interactions.

The statistical properties of the environmental measure, $\hat{\epsilon}_{j}$, are very similar when derived for the character final height from the parental average in the set of eight sowing date-density environments (Section 4) and in the set of 16 seasonal-locational environments of an earlier investigation (Bucio Alanis, 1966), as can be seen from the means, variances and ranges listed in table 12. Nevertheless, the edaphic, climatic and biological factors

TABLE 12

The statistical properties of the dependent environmental component, $\hat{\epsilon}_{\beta}$, derived for the character final height from the average of the two parents, varieties 1 and 5 , in each of the eight sowing datedensity environments and in each of 16 seasonal-locational environments

$\begin{array}{lcc} & \text { Sowing date-density } & \text { Seasonal-locational } \\ \text { Mean, } \hat{m} & 112.27 & 110.63 \\ \text { Variance } & 217.04 & 194.22 \\ \text { Range of } \hat{\epsilon}_{j} & -23.8 \text { to } 27.9 & -25.0 \text { to } 17.9\end{array}$

contributing to the differences within these two sets of environments are not expected to be the same. It is significant, therefore, that the reactions of varieties 1 and 5 and their $F_{1}$ in these two sets of environments for final height could not have been more different. In the sowing date-density set they display no significant interaction for this character (Section 4) while in the seasonal-locational set the same character shows significant genotypeenvironmental interactions, all of which are attributable to the linear component of the interactions (Bucio Alanis, Perkins and Jinks, 1969). This demonstrates in a more direct way than previously possible that it is the quality of the environments, as determined by the relative contributions of different physical and biological factors, that is responsible for the magnitude and properties of the genotype-environmental interactions and not the magnitude of the environmental values $\left(\hat{\epsilon}_{j}\right.$ or $\left.\hat{z}_{j}\right)$ themselves. In view of this conclusion it is difficult to maintain that the relative magnitudes of the interactions in a number of environments is merely a scalar effect arising from a simple relationship over the environments between mean performance and variance of the genotypes within each environment, as is so often assumed. It also highlights the problems that can arise when it is expedient to simula te the uncontrollable, naturally occurring environmental differences, e.g. between seasons, by some less demanding, systematically imposed treatments, e.g. sowing date differences within a season, in order to provide a practicable means of measuring and selecting for differences in sensitivity to seasonal differences (Perkins and Jinks, 1971a). There are clear dangers in doing so without information about either the degree of specificity of the reactions of 
the genotypes in the two kinds of environments or the nature of the basic factors responsible for the uncontrollable differences between these environments (Perkins, 1972).

Although varieties 1 and 5 do not interact with the sowing date-density environments, there is no doubt that the individual genes for which they differ do interact with these environments. Thus among the 82 inbred lines, obtained as a result of reassortment and recombinations of these genes, there are genotypes which show the greatest difference in linear sensitivity to these environments that have so far been observed. The absence of interactions in varieties 1 and 5 must, therefore, be attributed to a balance of genes which individually show above and below average sensitivity to the sowing datedensity environmental differences.

\section{REFERENCES}

BUCIO ALANIS, L. 1966. Environmental and genotype-environmental components of variability. I. Inbred lines. Heredity, 21, 387-397.

BUcio ALANIS, L., PERKINS, JEAN M., AND JINKs, J. L. 1969. Environmental and genotypeenvironmental components of variability. V. Segregatirg generations. Heredity, 24, $115-127$.

EAVES, L. J., AND BRUMPTON, R. J. 1972. Factors of covariation in Nicotiana rustica. Heredity, $29,151-175$.

FREEMAN, G. H., AND PERKINS, JEAN M. 1971. Environmental and genotype-environmental components of variability. VII. Relations between genotypes grown in different environments and measures of these environments. Heredity, 27, 15-23.

FRIPP, YVONNE. 1972. Genotype-environmental interactions in Schizophyllum commune. II. Assessing the environment. Heredity, 28, 223-238.

FRIPP, YVONNE, AND CATEN, C. E. 1971. Genotype-environmental interactions in Schizophyllum commune. I. Analysis and character. Heredity, 27, 393-408.

FRIPP, YVONNE, AND CATEN C. E. 1973. Genotype-environmental interactions in Schizophyllum commune. III. The relationship between mean expression and sensitivity to change in environment. Heredity, in press.

JINKS, J. L., AND JONES, R. MORLEY. 1958. Estimation of the components of heterosis. Genetics, 43, 63-85.

JINKS, J. L., AND PERKINS, JEAN M. 1969. The detection of linked epistatic genes for a metrical trait. Heredity, 24, 465-475.

MATHER, K., AND JINKS, J. L. 1971. Biometrical Genetics. Chapman and Hall Ltd., London. MATHER, K., AND VINES, A. 1952. The inheritance of height and flowering time in a cross of Nicotiana rustica. Quantitative Inheritance, ed. E. C. Reeve, pp. 49-80. C. H. Waddington, London.

PERKINS, JEAN M. 1972. The principal component analysis of genotype-environmental interactions and physical measures of the environment. Heredity, 29, 51-70.

PERKINS, JEAN M., AND JINKS, J. L. 1968a. Environmental and genotype-environmental components of variability. III. Multiple lines and crosses. Heredity, 23, 339-356.

PERKINS, JEAN M., AND JINKs, J- L. 1968b. Environmental and genotype-environmental components of variability. IV. Non-linear interactions for multiple inbred lines. Heredity, 23, 525-535.

PERKINS, JEAN M., AND JINKs, J. L. 1970. Detection and estimation of genotype-environmental, linkage and epistatic components of variation for a metrical trait. Heredity, 25, 157-177.

PERKINS, JEAN M., AND JINKS, J. L. 197 la. Analysis of genotype $\times$ environment interaction in triple test cross data. Heredity, 26, 203-209.

PERKINS, JEAN M., AND JINKS, J. Z. 1971b. Speciffcity of the interaction of genotypes with contrasting environments. Heredity, 26, 463-474.

SPEARMAN, C. 1904. The proof and measurement of association between two things. Amer. F. Psych., 15, 72-101.

yates, F., and cochran, w. G. 1938. The analysis of groups of experiments. F. Agric. Sci., $28,556-580$. 